\title{
Malakoplakia after kidney transplantation
}

\author{
Sang Jun Park ${ }^{1}$, Hojong Park ${ }^{1}$, Hong Rae Cho ${ }^{1}$, Kyung Sun Park ${ }^{2}$, Jongha Park ${ }^{2}$, Kyung Don Yoo ${ }^{2}$, Jong Soo Lee ${ }^{2}$ \\ ${ }^{1}$ Department of Surgery, Ulsan University Hospital, Ulsan, Korea \\ ${ }^{2}$ Division of Nephrology, Department of Internal Medicine, Ulsan University Hospital, Ulsan, Korea
}

Background: Malakoplakia is a rare pseudotumor that arises in the context of recurrent infections, particularly in the immunocompromised setting. We report a case of renal allograft parenchymal malakoplakia.

Methods: A 59-year-old woman had received cadaveric renal transplant due to diabetes in June 2018. Her creatinine remained relatively stable postoperatively. Two months after transplantation, she was treated with urinary tract infection (UTI). In March 2019, she had allograft biopsy for increasing creatinine. The biopsy identified acute T-cell mediated rejection and steroid pulse therapy was performed. In December 2019, she was hospitalized for right flank pain and pyuria. Her creatinine level was 1.9 mg/ dL. Ultrasound (US) renal Doppler and computed tomographic findings were likely to be hematoma or abscess in the perirenal area, and septated fluid collection was suspected. The lesion was connected to renal parenchyma. We thought that she was not the case of pyogenic abscess, so she had to undergo a biopsy because she needed to identify other causes. Biopsy results suggested malakoplakia and von Kossa stain with positive Michaelis- Gutmann bodies. The tissue culture found Escherichia coli, and this was treated with antibiotics. The dose of tacrolimus was reduced.

Results: After 1 month of hospitalization, she was discharged, and maintained oral antibiotics. In follow-up imaging, extent of lesion has increased, so we performed surgical resection and abscess drainage on February 3,2020 . Although there is still renal parenchymal involvement, the size is decreasing after 2 months of serial observation with US.

Conclusions: Malakoplakia is a rare condition, particularly kidney transplantation, where a differential diagnosis of recurrent UTI with graft dysfunction and the presence of pseudotumoral lesions should be considered. The treatment is reduction in immunosuppression; a good response is achieved with medical therapy using long-term antibiotic treatment. However, early surgical treatment must be considered for refractory cases. 